\title{
Funções de afilamento para uma comunidade arbórea na floresta estadual do Amapá (flota/AP)
}

\author{
Tapering functions for an arboreal community in the state forest of amapá \\ (flota / AP)
}

\author{
Rodrigo Galvão Teixeira de Souza ${ }^{1}$, Hugo Leonardo Pires e Pires², Anthoinny Vittória dos \\ Santos Silva ${ }^{3}$, Gabriel Victor Caetano carvalho Liarte ${ }^{4}$, Robson Borges de Lima ${ }^{5}$, Jadson \\ Coelho de Abreu
}

${ }^{1}$ Acadêmico de Engenharia Florestal da Universidade do Estado do Amapá, Brasil. E-mail: rodrigosouza55@hotmail.com * Autor para correspondência

${ }^{2}$ Engenheiro Florestal pela Universidade do Estado do Amapá, Brasil. E-mail: leonardopires87@hotmail.com

${ }^{3}$ Acadêmica de Engenharia Florestal da Universidade do Estado do Amapá, Brasil. E-mail: vittoria.sntsilva@gmail.com

${ }^{4}$ Acadêmico de Engenharia Florestal da Universidade do estado do Amapá, Brasil. E-mail: gv326766@gmail.com

${ }^{5}$ Engenheiro Florestal, Doutor em Ciências Florestais pela Universidade Federal Rural de Pernambuco, Docente da Universidade do Estado do Amapá- UEAP. Brasil. E-mail: rbl florestal@yahoo.com.br

${ }^{6}$ Engenheiro Florestal, Doutor em Ciência Florestal pela Universidade Federal de Viçosa, Docente da Universidade do Estado do Amapá-UEAP. Brasil. E-mail: jadsoncoelhoabreu@hotmail.com

\section{Palavras-chave}

Modelo

Volume

Regressão

Amazônia

\section{Keywords}

Mode

Volume

Regression

Amazônia
A região amazônica se destaca pela intensa riqueza dos recursos florestais, e com essa grande variação de diversidade, há necessidade de se realizar planejamentos rigorosos visando a sustentabilidade proporcionando um ciclo produtivo e constante. Com isso, o objetivo do presente trabalho foi ajustar, avaliar e selecionar funções de afilamento para uma comunidade arbórea no Estado do Amapá. O trabalho foi realizado em uma das maiores unidades de conservação do Estado - FLOTA/AP, mais precisamente no Modulo 4, localizado entre os municípios de Calçoene e Oiapoque. Para o ajuste das funções de afilamento, seguiram-se algumas metodologias pertinentes à literatura sendo coletadas por árvore as seguintes variáveis: Diâmetro à altura do peito, altura comercial e os diâmetros com casca em 10\%, 20\%, 30\%, 40\%, 50\%, 60\%, 70\%, 80\%, 90\% e $100 \%$ da altura comercial, totalizando 12 pontos ao longo do tronco das espécies estudadas. Foram ajustados os modelos de Kozak, Polinômio de Quinto Grau, Garcia e Bennett e Swindel. Para avaliar a acuracidade das funções de afilamento, foram calculados as estatísticas erro padrão de estimativa em porcentagem (Syx\%), o coeficiente de determinação ajustado ( $R^{2}$ ajs.), e a distribuição percentual dos resíduos, por meio do Software R 3.5. O modelo de afilamento mais indicado para a estimativa do diâmetro das árvores foi o Bennett e Swindel, pois demonstrou superioridade nas estatísticas comparativas, já o modelo Polinômio de quinto grau foi o menos indicado para as espécies em estudo.

The Amazon region stands out for its intense richness of forest resources, and with this wide variation in diversity, there is a need to carry out rigorous planning aiming at sustainability providing a productive and constant cycle. Thus, the objective of the present work was to adjust, evaluate and select tapering functions for a arboreal community in the Amazon region of Amapá State. The work was carried out in one of the largest conservation units in the state - FLOTA / AP, more precisely in module 4, located between the municipalities of Calçoene and Oiapoque. To adjust the tapering functions, some pertinent literature methodologies were followed. The following variables were collected by tree: Diameter at breast height, commercial height and bark diameters at 10\%, 20\%, 30\%, 40\%, 50\%, 60\%, 70\%, 80\%, 90\% and 100\% of commercial height, totaling 12 points along the trunk of the studied species. Kozak, Fifth Grade Polynomial, Garcia and Bennett and Swindel models were adjusted. To assess the accuracy of the taper functions, the standard error percent estimation statistics (Syx\%), the adjusted determination coefficient $\left(R^{2} a j s.\right)$, And the percentage distribution of residuals were calculated using the Software $R$ 3.5. The best tapering model for estimating tree diameter was Bennett and Swindel, as it showed superiority in comparative statistics, while the fifth degree polynomial model was the least indicated for the species under study.

\section{INTRODUÇÃO}

A região amazônica se destaca pela intensa riqueza dos recursos florestais, e com essa grande riqueza e diversidade, há necessidade de se realizar planejamentos rigorosos visando a sustentabilidade, proporcionando um ciclo produtivo e constante. O Estado do Amapá ainda se encontra com sua maior parte territorial preservada, sendo 
considerado como o Estado mais preservado do Brasil, apresentando as menores taxas de desmatamento devido as grandes áreas de unidades de conservação de uso sustentável (NASCIMENTO, 2012).

Com destaque para a Floresta Estadual do Amapá (FLOTAAP), criada pela Lei no 1.028, de 12 de julho de 2006 (AMAPÁ, 2006) com área aproximada de 2,3 milhões de hectares, englobando parte de 10 municípios do Estado é dividida em quatro módulos (IEF, 2014). Com a realização do plano de manejo, esta será submetida à concessão para que seus produtos florestais madeireiros e não-madeireiros passem a ser utilizados de forma sustentável.

As atividades florestais sem a presença de um planejamento vêm crescendo nos últimos anos, causando crescentes desmatamentos de extensas áreas de cobertura vegetal na Amazônia, onde espécies raras têm sido extintas e diversos habitats modificados pelas ações antrópicas, o que afeta diretamente na resistência e resiliência dos ecossistemas (SILVA et al., 2015).

Por meio da elaboração de um bom planejamento, possibilita-se alternativas e prioridades no uso adequado da madeira, assim também a definição e meios de estratégias atuais contribuirão para os resultados satisfatórios no futuro, bem como as diminuições nos danos ambientais. É de suma importância se atentar para tais fatos, pois estes são extremamente marcantes na atividade florestal que apresenta longos ciclos de produção (CAMPOS et al., 2013).

Miguel et al. (2011) ressaltam que é necessário utilizar técnicas de quantificação e qualificação desses produtos, entre as quais se têm os modelos de afilamento, que são ferramentas estatísticas que possibilita representar o perfil do tronco das árvores através de cálculos obtendo seu volume total e comercial entre pontos quaisquer, bem como seu diâmetro em qualquer altura especificada e a altura total ou a que se encontra em um determinado diâmetro, e segundo Nicoletti (2017), o uso de funções de afilamento proporcionam uma otimização dos recursos florestais.

Com isso, este trabalho tem como objetivo ajustar e definir os melhores modelos de afilamento para representar o perfil do fuste das espécies nativas de interesse comercial, visando um melhor aproveitamento da madeira na floresta localizada no módulo IV da FLOTA-AP.

\section{MATERIAL E MÉTODOS}

\section{Área de estudo}

O presente estudo foi desenvolvido na região do Módulo IV da Floresta Estadual do Amapá (FLOTA/AP), Distante 630 $\mathrm{km}$ da capital Macapá, o módulo IV possui uma área de aproximadamente $9.739,7 \mathrm{~km}^{2}$, localizado na região norte do Estado, Conforme a classificação de Köppen, o clima da região é classificado como Amw', apresentando uma estação chuvosa de novembro a junho e uma estação seca de julho a outubro (IEPA, 2008), a temperatura apresenta uma baixa amplitude térmica, de $24,8 \pm 0,15{ }^{\circ} \mathrm{C}$ no mês mais frio a 26,9 $\pm 0,10 \cong \mathrm{C}$ no mês mais quente (Figura 1 ).

Figura 1. Mapa de localização da área de estudo do modulo IV Próximo ao rio Cassiporé.

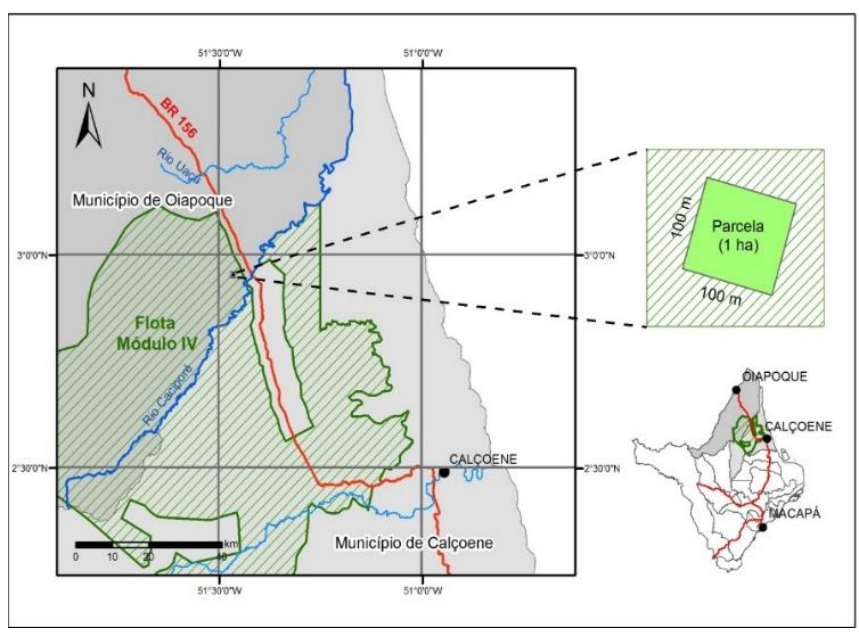

A cobertura vegetal da área de estudo caracteriza-se por uma típica floresta ombrófila densa de terra firme, seu dossel está entre 30 e $35 \mathrm{~m}$ de altura, possuindo espécies emergentes que podem atingir até $45 \mathrm{~m}$, indicando a formação da tipologia florestal de submontana (IEPA, 2008).

\section{Inventário da área estudada}

O método de amostragem a ser empregado foi de área fixa, representado por uma parcela quadrada (100 m x $100 \mathrm{~m}$ ) equivalendo a uma área de um hectare, sendo posteriormente dividida em subparcelas (10 m x $10 \mathrm{~m}$ ), para facilitar o inventario e a localização das árvores (Figura 1). Dentro da parcela (100 m x $100 \mathrm{~m}$ ) foram mensuradas e coletadas por árvore as seguintes variáveis: Circunferência ao nível de 1,30 metros do solo, com uma fita métrica, posteriormente dividindo por $\pi$ para obter o valor de diâmetro (DAP cm) num nível de inclusão de DAP $\geq 40 \mathrm{~cm}$, coletou-se também a altura comercial e altura total por meio de hipsômetro Trupulse. E paralelamente a isso, foi realizado ainda no campo, um reconhecimento preliminar desses indivíduos por um parataxonomista, e os nomes científicos pesquisados em literatura pertinente.

Após o inventário foi realizado o corte de todas as árvores que estavam dentro do nível de inclusão e após o

corte foi executado as medições ao longo de cada árvore seguindo a mesma metodologia de Ramos (2012), medindo- 
se as circunferências para obter os diâmetros com casca em 12 pontos ao longo do tronco comercial (ponto de corte até a base da copa), da seguinte forma:

- Ponto de corte considerado como início da medição de altura comercial $(0,0 \mathrm{~m})$;

- 1,3 m ou Diâmetro à Altura do Peito (d1,3);

- $10 \%, 20 \%, 30 \%, 40 \%, 50 \%, 60 \%, 70 \%, 80 \%, 90 \%$ e $100 \%$ da altura comercial.

\section{Ajuste das funções de afilamento}

Para a determinação do ajuste da equação de Taper para o tronco comercial, foram selecionadas quatro funções de afilamento utilizando-se as técnicas de regressão linear de Kozak et al. (1969), Polinômio de quinto grau (SCHÖEPFER, 1966), Garcia et al. (1993), e Bennett e Swindel (1972), utilizadas também por Ramos (2012) e Costa et al., (2016) sendo as funções consideradas como as mais utilizados para essa finalidade e que em outros trabalhos apresentaram um bom ajuste principalmente quando se trata de espécies florestais nativas. Estes modelos estão descritos abaixo conforme mostrado na tabela 1.

\section{Parâmetros estatísticos de avaliação}

Para avaliar a acuracidade das funções de afilamento foram calculadas as estatísticas: Erro Padrão de Estimativa Real em porcentagem (Syx\%), que indica o quão próximo os valores estimados estão dos valores observados quanto à curva de regressão e quanto mais próximo de " 0 " (zero), melhor será a equação; Coeficiente de Determinação Ajustado (R2ajs.), que indica o quanto a variável dependente é explicada pelas independentes ou o quanto da variação é explicada pela regressão, sendo que quanto mais próximo de "100 \%" (cem por cento), melhor é a equação; e a Distribuição percentual dos resíduos, indica se a estimativa é satisfatória ao longo da linha de regressão, se não há tendenciosidades em nenhum de seus segmentos e se a equação é realmente apropriada para tal fim (LEITE et al., 2006). Os cálculos foram realizados utilizando-se o Software $\mathrm{R}$ 3.5.

$$
\begin{aligned}
& \text { Syx }=\left(\frac{\sqrt{\frac{\sum_{i=0}^{n}(y i-\hat{y} i)^{2}}{n-p-1}}}{\bar{y}}\right) \cdot 100 \\
& R^{2} \text { ajs }=\left(1-\left(\frac{\text { SQres }}{\text { SQt }}\right) *\left(\frac{n-1}{n-p}\right)\right) \cdot 100
\end{aligned}
$$

\section{RESULTADOS E DISCUSSÃO}

Na Tabela 2 observa-se a relação de todas as espécies utilizadas para este estudo. Sendo amostrados 53 indivíduos, distribuídos em 17 famílias botânicas, e 32 espécies.

Analisando a quantidade dessas espécies (32), se confirma as alegações apresentadas por Andrade e Manzatto (2014) relacionadas a vasta biodiversidade do bioma amazônico.

Observando de modo geral o conjunto dos indivíduos, a variável diâmetro obteve médias consideradas elevadas, sendo que o diâmetro mínimo para o conjunto dos dados foi de 24,51 cm, já para o diâmetro máximo foi 241,92 cm, denominado uma floresta bastante heterogênea e em diferentes estágios de sucessão. No trabalho de Silva et al. (2020) realizado em matas de terra firme no estado do Pará a altura mínima encontrada para os indivíduos arbóreos foi de

Tabela 1. Modelos matemáticos testados para expressar o perfil dos troncos das árvores amostradas; № = número do modelo; di = diâmetro tomado nas diferentes alturas hi ao longo do tronco, em centímetros; DAP = diâmetro a altura do peito, em centímetros; hi = altura até uma seção i do fuste, em metros; $\mathrm{H}=$ altura comercial, em metros; $\mathrm{Bi}=$ parâmetros a serem estimados.

\begin{tabular}{lcc}
\hline $\mathbf{N}^{\mathbf{0}}$ & MODELO & AUTOR \\
\hline 1 & $\left(\frac{d i}{D A P}\right)^{2}=\beta_{o}+\beta_{1}\left(\frac{h i}{H}\right)+\beta_{2}\left(\frac{h i}{H}\right)^{2}$ & Kozak et al. (1969) \\
$2 \quad \frac{d i}{D A P}=\beta_{o}+\beta_{1}\left(\frac{h i}{H}\right)+\beta_{2}\left(\frac{h i}{H}\right)^{2}+\beta_{3}\left(\frac{h i}{H}\right)^{3}+\beta_{4}\left(\frac{h i}{H}\right)^{4}+\beta_{5}\left(\frac{h i}{H}\right)^{5}$ & Polinômio de 5 \\
$3\left(\frac{d i}{D A P}\right)^{2}=\beta_{o}+\beta_{1} \sqrt{\frac{h i}{H}}+\beta_{2}\left(\frac{h i}{H}\right)$ & grau \\
4 & $\frac{d i}{D A P}=\beta_{o}+\beta_{1}\left(\frac{H}{H+h i}\right)+\beta_{2}\left(\frac{H}{H+h i}\right)^{2}+\beta_{3}\left(\frac{H}{H+h i}\right)^{3}+\beta_{4}\left(\frac{H}{H+h i}\right)^{4}$ & Gepfer, 1966) \\
& & Gennett e Swindel \\
\end{tabular}


Tabela 2. Quantidade de árvores amostradas por espécie e família.

\begin{tabular}{|c|c|c|c|}
\hline Nome Popular & Nome científico & Família & Quantidade \\
\hline Abiu folha & Ecclinusa abbreviata Ducke & Sapotaceae & 1 \\
\hline Abiurana & Pouteria macrophylla (A. DC) & Sapotaceae & 3 \\
\hline Acariquara & Minquartia guianensis Aubl. & Olacaceae & 4 \\
\hline Andiroba & Carapa guianensis Aubl. & Meliaceae & 3 \\
\hline angelim pedra & Hymenolobium excelsun Ducke & Fabaceae & 1 \\
\hline Araracanga & Aspidosperma desmanthum Benth ex. Muell. Arg. & Apocynaceae & 1 \\
\hline axuá & Edopleura Uchi (Huber) Cuatrec. & Humiriaceae & 1 \\
\hline Breu branco & Protium heptaphyllum Aubl. & Burseraceae & 6 \\
\hline Carapanaúba & Aspidosperma carapanauba Pichon & Apocynaceae & 1 \\
\hline casca de vidro & Pouteria pachycarpa Pires & Sapotaceae & 1 \\
\hline cupiúba & Goupia glabra Aubl & Celastraceae & 3 \\
\hline faveira & Parkia decussada Ducke & Fabaceae & 2 \\
\hline ínga vermelho & Heterophylla Willd. & Fabaceae & 1 \\
\hline Louro vermelho & Licaria canella (Meissner) Kosterm & Lauraceae & 1 \\
\hline Macucú & Licania heteromorpha Benth. & Chrysobalanaceae & 1 \\
\hline mamorana & Paquiara aquatica Aubl. & Malvaceae & 1 \\
\hline Mandioqueira & Qualea belemensis (Staflem) Marc.-Berti. & Vochysiaceae & 2 \\
\hline Marupá & Simaruba amara Aubl. & Simaroubaceae & 1 \\
\hline Matamata & Eschweilera apiculata (Miers) Smith & Lecythidaceae & 2 \\
\hline Melancieiro & Alexa grandiflora Ducke & Fabaceae & 1 \\
\hline meraúba & Mouriri duckeanoide Morley & Muiraúba & 1 \\
\hline mututirana & Pterocarpus officinallis Jack. & Fabaceae & 1 \\
\hline Parapará & Jacaranda copaia (Aubl) D. Don & Bignoniaceae & 1 \\
\hline Paricá & Schyzolobium amazonicum (Huber) Ducke & Fabaceae & 3 \\
\hline quaruba & Vochysia maxima Ducke & Vochysiaceae & 2 \\
\hline Sorva & Couma utilis (Mart.) M. Arg. & Apocynaceae & 1 \\
\hline Sucupira Amarela & Bowdichia nitida Spruce ex Benth. & Fabaceae & 1 \\
\hline Sucupira preta & Bowdichia virgilioides & Fabaceae & 1 \\
\hline Tauarí & Couratari guianensis Aubl. & Lecythidaceae & 1 \\
\hline Taxi vermelho & $\begin{array}{l}\text { Sclerolobium melanocarpum Ducke (J. F. Guimel.) } \\
\text { Exell. }\end{array}$ & Fabaceae & 1 \\
\hline Ucuubarana & Iryanthera grandis Ducke & Myristicaceae & 2 \\
\hline Urucurana & Sloanea guianensis & Elaeocarpaceae & 1 \\
\hline TOTAL & 32 & 17 & 53 \\
\hline
\end{tabular}

8,00 metros e máxima de 36,20 metros, demostrando métricas similares a este trabalho, onde foi observado a mínima e máxima altura de 7,57 e 29,70 metros, respectivamente.

Após o ajuste dos quatro modelos de afilamento para diâmetro estimado, foram realizadas as análises estatísticas para avaliar sua precisão por meio de coeficiente de determinação ajustado $\left(R^{2}\right)$ e erro padrão da estimativa (Syx\%), (Tabela 3).

Os modelos testados mostraram bons resultados em relação as estatísticas de ajuste, entretanto, por se tratar de uma comunidade arbórea composta por diversas espécies, nota-se uma grande variação do comprimento e diâmetro do fuste das árvores ao se comparar com uma floresta plantada 
Tabela 3. Resultados dos ajustes das equações de afilamento para o conjunto dos indivíduos.

\begin{tabular}{|c|c|c|c|c|c|c|c|c|}
\hline \multicolumn{9}{|c|}{ Coeficientes/Parâmetros estimados } \\
\hline Modelo & $\beta_{0}$ & $\beta_{1}$ & $\beta_{2}$ & $\beta_{3}$ & $\beta_{4}$ & $\beta_{5}$ & $\mathrm{R}_{\text {ajs }}^{2}$ & $\mathrm{~S}_{\mathrm{yx}} \%$ \\
\hline Bennett \& Swindel & 11,660 & $-62,376$ & 132,188 & $-122,821$ & 42,564 & & 86,49 & 18,01 \\
\hline Garcia & 1,320 & $-0,768$ & $-0,000$ & & & & 85,52 & 18,89 \\
\hline Kozak & 1,319 & $-2,041$ & 1,487 & & & & 85,57 & 18,86 \\
\hline Pol. do $5^{\circ}$ grau & 1,177 & $-1,494$ & 0,301 & 7,783 & $-13,371$ & 6,445 & 84,65 & 21,23 \\
\hline
\end{tabular}

Figura 2. Distribuição gráfica dos resíduos em porcentagem para todas as espécies com os 4 modelos estudados.
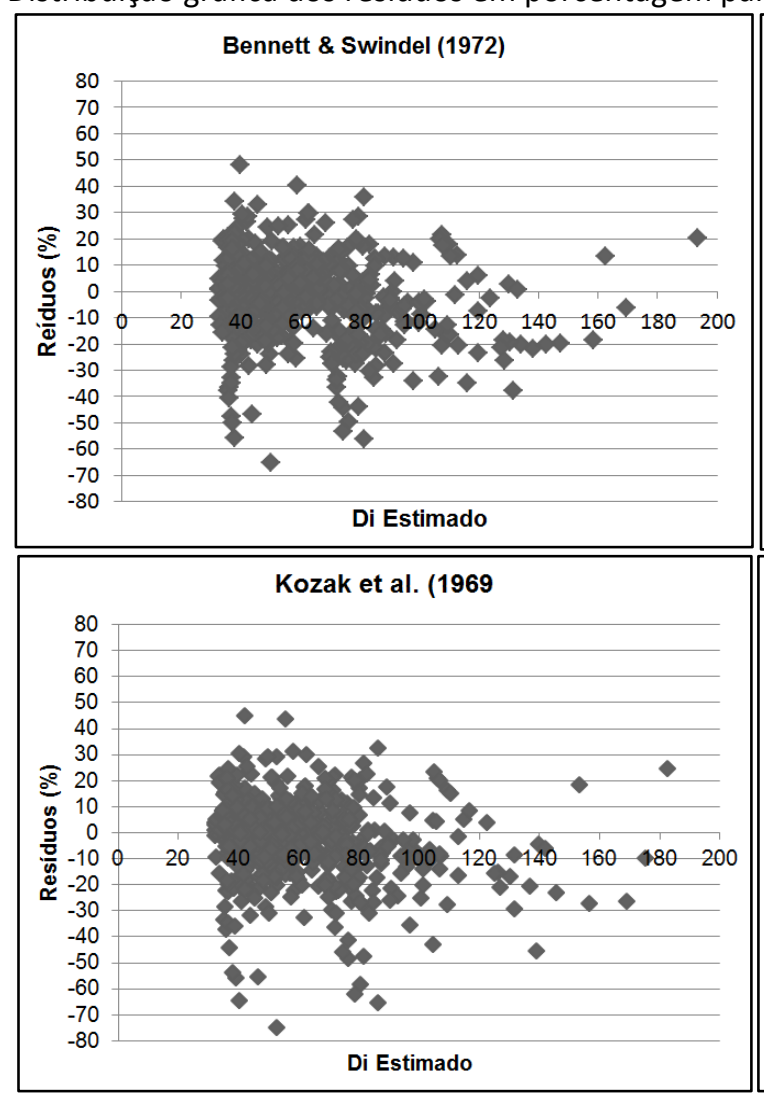

de Eucalyptus sp. ou Pinus sp., nos trabalhos sobre afilamento do fuste de (Farias et al., 2019; Silva et al., 2018) realizados em florestas de Eucalyptus Grandis e Pinus Elliottii foi ajustado várias funções de taper, entre elas o modelo de kozak et al. (1969), Garcia et al. (1993), entre outros, observase um ( $R^{2}$ ajs) que vária de 0,90 a 0,96 e (Syx\%) de 8,3 a 10,8, desse modo, é possivelmente explicado a baixa do (R2ajs) e aumento do (Syx\%) em funções ajustadas para florestas nativas.

As equações ajustadas demostraram muita similaridade nos valores do erro padrão da estimativa (Syx\%), entretanto, o modelo Polinômio do 5 o grau apresentou maiores valores do (Syx\%), sendo ele, o modelo mais inferior. Com base nessas estatísticas, constata-se uma pequena vantagem para o Modelo de Bennett \& Swindel com erro padrão da estimativa (Syx\%) de $18,01 \%$ e coeficiente de determinação ( $\left.R^{2} a j\right)$ de $86,49 \%$.

Para escolher a equação que melhor se ajustou às
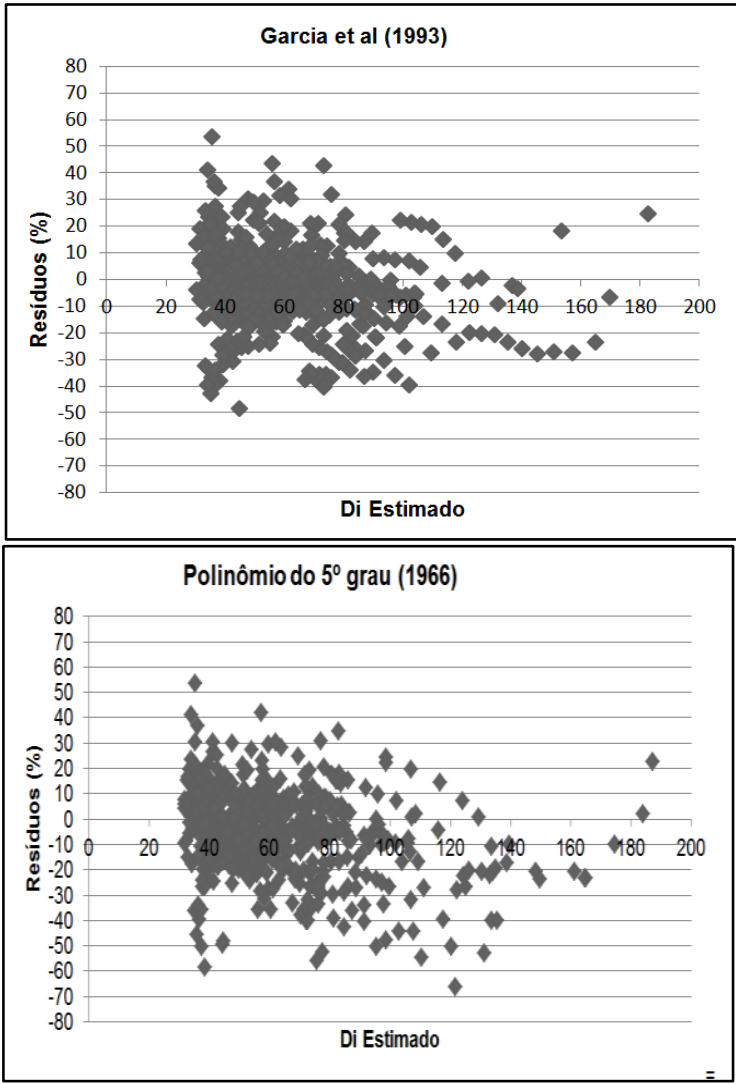

condições apresentadas no trabalho, os gráficos de resíduos foram analisados, juntamente com os erros-padrão de estimativa e com os coeficientes de determinação ajustados, de acordo com Silva et al. (2018) quando um modelo demonstra uma alta dispersão residual chegando a mostrar tendenciosidades é correto afirmar que o modelo está tendo dificuldade na estimativa, deste modo ao observar os gráficos de dispersão dos resíduos na Figura 2, percebe-se que todos os modelos apresentam dispersões residuais agravadas nos diâmetros mínimos, destarte as estimativas tendem a superestimação ou subestimação com maior frequência nesses pontos. Ao levar em conta a estimativa dos diâmetros maiores, percebe-se um erro menor na predição, que, é possivelmente explicado, pois existe uma relação de desproporcionalidade entre a estimativa de diâmetros pequenos e diâmetro de valores maiores, quando um modelo superestima ou subestima 1 centímetro em um diâmetro grande, esse erro pode ser considerado relativamente 
pequeno, entretanto, ao se tratar de um diâmetro pequeno, o erro de 1 centímetro pode ser classificado como um erro alto.

O modelo de Bennett \& Swindel obteve um ajuste superior quando comparado com os demais, uma vez que mostrou menores discrepâncias nas estimativas e sua dispersão residual está tendendo a homogeneidade. Apesar de ter apresentado estatísticas de precisão satisfatória o modelo Polinômio de quinto grau (SCHÖEPFER, 1966) foi o de maior inferioridade, tendendo a superestimar e subestimar os diâmetros ao longo do fuste para a parte superior, entretanto não é descartado para representar o afilamento do fuste das espécies estudadas.

\section{CONCLUSÃO}

De acordo com as estatísticas de acuracidade, o modelo de afilamento mais indicado para a estimativa do volume das árvores contidas nessa comunidade florestal, foi o modelo de Bennett e Swindel (1972), já a equação do modelo Polinômio de quinto grau (SCHÖEPFER, 1966) foi a menos indicada para as espécies em estudo, pois demonstrou inferioridade nas estatísticas de escolha e apresentou a distribuição gráfica do resíduo tendenciosa.

\section{REFERÊNCIAS}

ANDRADE, R. T. G. \& MANZATTO, Â. G. A Insuficiência de Políticas Públicas Nacionais Pró-Biodiversidade Amazônica. Revista Gestão \& Políticas Públicas, 4(2), 219239, 2014.

AMAPÁ; Lei $n^{\circ} 1.028$, de 12 de julho de 2006. Dispõe sobre a criação e gestão da Floresta Estadual do Amapá, e dá outras providências. Diário Oficial do Amapá, Macapá 28 junho de 2006. <Disponível em http://www.ief.ap.gov.br>. Acesso em 16 de janeiro de 2015.

ANDRADE, R.T. G.; MANZATTO, Â.G. A Insuficiência de Políticas Públicas Nacionais Pró-Biodiversidade Amazônica. Revista Gestão \& Políticas Públicas, v. 4, n. 2, p. 219-239, 2014.

AZEVEDO, L. S. Análise florística e da estrutura horizontal de uma floresta de terra firme na comunidade de Lindóia, no município de Itacoatiara-AM. Itacoatiara: UEA, 2017. Monografia (Graduação em Engenharia Florestal), Faculdade de Engenharia Florestal, Universidade Estadual do Amazonas, 2017.

CAMPOS, B.P.F.; BINOTI, D.H.B.; SILVA, M.L.; LEITE, H.G.; BINOTI, M.L.M.S. Conversão de árvores em multiprodutos da madeira utilizando programação inteira. Revista árvore. Viçosa-MG, vol.37 n.5, p.881-887. 2013.

COSTA, E. A.; FINGER, C. A. G.; SCHNEIDER, P. R.; HESS, A. F.
Função de afilamento e sortimentos de madeira para Araucaria angustifolia. Ciência florestal, 26(2), 523-533, 2016.

FARIAS, K. J.; NICOLETTI, M. F.; AGUIAR, N. S. Quantificação do volume total por meio de modelos volumétricos e de afilamento em Eucalyptus grandis W. Hill ex Maiden. Revista de Ciências Agroveterinárias, 18(1), 33-40, 2019.

INSTITUTO DE PESQUISAS CIENTÍFICAS E TECNOLOGICAS ESTADO DO AMAPÁ (Macapá). Macrodiagnóstico do estado do Amapá, primeira aproximação do ZEE. Amapá, 139 p., 2008.

INSTITUTO ESTADUAL DE FLORESTAS DO AMAPÁ (IEF). Plano de manejo da Floresta Estadual do Amapá. Resumo Executivo da FLOTA/AP. Governo do Estado do Amapá. Secretaria de Estado do meio ambiente - SEMA. 60 p. Macapá-AP: 2014.

LEITE, H. G.; GAMA, J. R. V.; CRUZ, J. P., SOUZA, A. L. Função de afilamento para Virola surinamensis (Roll.) Warb. Revista Árvore, v. 30, n. 1, p. 99-106. Viçosa-MG, 2006.

MIGUEL, E.P.; MACHADO, S.A.; FILHO, A.F.; ARCE, J.E. Modelos polinomiais para representar o perfil e o volume do fuste de Eucalyptus urophylla na região norte do estado de Goiás. Floresta, Curitiba, PR, v. 41, n. 2, p. 355-368, abr./jun. 2011.

NASCIMENTO, G.D.; Comercialização e uso da diversidade de flora do módulo 4 da floresta estadual do amapá pela comunidade do carnot, Calçoene, amapá, brasil. 2012. 82 f. Dissertação (Mestrado em Biodiversidade Tropical) Universidade Federal do Amapá, Empresa Brasileira de Pesquisa Agropecuária, IEPA / CI - BRASIL, Macapá-AP, 2012.

NICOLETTI M.F. Modelagem não linear mista e método bivariado para sortimento da produção de Pinus taeda $L$. Tese (Doutorado em Engenharia Florestal). Curitiba: UFPR. $118 p, 2017$.

RAMOS, M. Comparação de métodos para estimar o volume comercial em floresta ombrófila densa no Estado do Amazonas. 54 f. Dissertação (Mestrado em Ciências Florestais) - Programa de Pós-Graduação em área de concentração em Manejo Sustentável dos Recursos Florestais, Universidade Estadual do Centro-Oeste, IratiPR, 2012.

SILVA, K. E.; SOUZA, C. R.; AZEVEDO, C. P.; ROSSI, L. M. B. Dinâmica florestal, estoque de carbono e fitossociologia de uma floresta densa de terra-firme na Amazônia Central. Embrapa Amazônia Ocidental-Artigo em periódico indexado (ALICE), 2015.

SILVA, M. V. C.; ANDRADE, R.Y.; LIMEIRA, M. M. C.; COELHO, M. C. B.; SANTOS, A. F.; GIONGO, M.; ERPEN, M. L. Uso de redes neurais artificiais e modelos de regressão para estimar volume de espécies nativas em Portel, Pará-Brasil. 
Revista Forestal Mesoamericana Kurú, 17(40), 2020.

SILVA, S. A.; FERRARI, L. S.; MOREIRA, D.; LANSSANOVA, L. R.; ANSOLIN, R. D.; CIARNOSCHI, L. D.; NAKAJIMA, N. Y. Funções de afilamento para pinus elliottii engelm. proveniente de condução de regeneração natural. BIOFIX Scientific Journal, 3(1), 130-136, 2018.

Submissão: 20/02/2020

Aprovado para publicação: 04/03/2020 